Appl. Set-Valued Anal. Optim. 2 (2020), No. 3, pp. 329-337

Available online at http://asvao.biemdas.com

https://doi.org/10.23952/asvao.2.2020.3.07

\title{
A NORMAL-SUBGRADIENT ALGORITHM FOR FIXED POINT PROBLEMS AND QUASICONVEX EQUILIBRIUM PROBLEMS
}

\author{
LE HAI YEN ${ }^{1}$, LE DUNG MUU ${ }^{1,2 *}$ \\ ${ }^{1}$ Institute of Mathematics, VAST, Hanoi, Viet Nam \\ ${ }^{2}$ TIMAS, Thang Long University, Viet Nam
}

\begin{abstract}
We propose an algorithm for approximating a solution of a quasiconvex, para-monotone equilibrium problem, which is also a fixed point of a nonexpansive operator. The proposed algorithm is a combination between the projection one with the research direction being the normal subgradient of the quasiconvex bifunction for the equilibrium problem and the Krasnoselskii - Man iterative scheme for the fixed point one. Convergence of the algorithm is analyzed and some special models of the problem are presented.
\end{abstract}

Keywords. Equilibrium problem; Fixed point; Quasiconvex equilibrium problem; Normal subgradient algorithm, Krasnoselskii - Man scheme

\section{INTRODUCTION}

In this paper, we are concerned with the equilibrium problem stated as

$$
\text { finding } x^{*} \in C \text { such that } f\left(x^{*}, y\right) \geq 0, \quad \forall y \in C,
$$

where $C$ is a nonempty closed convex subset in $\mathbb{R}^{n}$, and $f: C \times C \rightarrow \mathbb{R} \cup\{+\infty\}$ is a bifunction such that $f(x, y)<+\infty$ for every $x, y \in C$. The inequality in problem (EP) was first used in [12] for the convex noncooperative game theory. The first result on the existence of solutions of (EP) is due to Fan [6], where this problem was called a minimax inequality. The name equilibria was first used in [11]. After the appearance of the paper by Blum and Oettli [3], problem (EP) has attracted much attention of many authors and a lot of algorithms have been developed for solving the problem, where the bifunction $f$ has some monotonic properties. Comprehensive reference lists on theory and algorithms for the equilibrium problem can be found in the monographs $[4,9]$.

An interesting point of this problem is that, despite its simple formulation, it contains many problems, such as, variational inequality problems, minimax problems, saddle point problems, Kakutani fixed point problems, Nash equilibrium problems, and some others as special cases; see, e.g., $[4,9,19]$.

Let $T: C \rightarrow C$ be a mapping on $C$. Recall that $T$ is said to be nonexpansive if and only if $\| T x-$ $T y\|\leq\| x-y \|$ for every $x, y \in C$. The problem of finding a fixed point of $T$ is a fundamental

${ }^{*}$ Corresponding author.

E-mail addresses: lhyen@math.ac.vn (L.H. Yen), ldmuu@math.ac.vn (L.D. Muu).

Received April 15, 2020; Accepted October 28, 2020.

(C)2020 Applied Set-Valued Analysis and Optimization 
research in the fixed point theory. A lot number of methods for solving this problem has been developed; see, e.g., [5] and the references therein.

In this paper, we focus on the problem of finding a point in $C$ such that it is a solution of problem (EP), which is also a fixed point of $T$. This problem can be formulated as

$$
\text { finding } x^{*} \in C \text { such that } f\left(x^{*}, x\right) \geq 0, \quad \forall x \in C \text { and } x^{*}=T\left(x^{*}\right) .
$$

Earlier results on this problem are due to Tada and Takahashi [17], and Takahashi and Takahashi [18], where the regularization method and the viscosity method were used to obtain weak and strong convergence of their algorithms for this problem. Since then, various algorithms, which are based on the projection method and the Krasnoselskii-Mann iteration, have been developed to obtain a solution of the equilibrium problem, which is also a fixed point of a nonexpansive mapping; see, e.g., [1, 2, 16, 13]. It should be noticed that all existing algorithms fail to apply to problem (FEP) when the bifunction $f$ is quasiconvex with respect to its second variable.

In this paper, we propose an algorithm for solving problem (FEP), where the bifunction $f$ may be quasiconvex. The proposed algorithm is a combination of the projection for (EP) and the Krasnoschelski-Mann iteration for fixed points of nonexpansive mappings. In order to handle the quasiconvexity, we use a normal subgradient as the direction at each iteration. Convergence of the algorithm is proved and some special cases of the problem are considered.

The organization of the paper is as follows. In Section 2, we recall some preliminaries on quasiconvex functions and their subdiffrentials. Section 3 is devoted to the analysis of the convergence of a normal subgradient algorithm. The special cases of problem (FEP) are considered as the practical models in Section 4. Finally, Section 5 ends this paper with a conclusion.

\section{PRELIMINARIES}

First of all, let us recall the definitions of the quasiconvex function and its star-subdifferential that will be used in our algorithm.

Definition 2.1. A function $\varphi: \mathbb{R}^{n} \rightarrow \mathbb{R} \cup\{+\infty\}$ is said to be quasiconvex on a convex subset $Y$ of $\mathbb{R}^{n}$ if and only if, for every $x, y \in Y$ and $\lambda \in[0,1]$,

$$
\varphi[(1-\lambda) x+\lambda y] \leq \max [\varphi(x), \varphi(y)] .
$$

It is easy to see that $\varphi$ is quasiconvex on a convex set $Y$ if and only if the level set $\{x \in Y$ : $\varphi(x)<\alpha\}$ on $Y$ of $\varphi$ at $x$ is convex for every $\alpha \in \mathbb{R}$.

The star-sudifferential of $\varphi$ (see, e.g., [15]) is defined as

$$
\partial^{*} \varphi(x):=\left\{g \in \mathbb{R}^{n}:\langle g, y-x\rangle<0 \forall y \in L_{\varphi}(x)\right\},
$$

where $L_{\varphi}(x):=\left\{y \in R^{n}: \varphi(y)<\varphi(x)\right\}$ is the level set of $\varphi$ at the level $\varphi(x)$. Clearly, if $\bar{L}_{\varphi}(x)$ is the closure of $L_{\varphi}(x)$, then

$$
\partial^{*} \varphi(x):=\left\{g \in \mathbb{R}^{n} ;\langle g, y-x\rangle \leq 0 \forall y \in \bar{L}_{\varphi}(x)\right\} .
$$

Hence $\partial^{*} \varphi(x) \equiv \mathbb{R}^{n}$ if $x$ is a minimizer of $\varphi$ over $\mathbb{R}^{n}$, and if $\varphi$ is continuous on $\mathbb{R}^{n}$, then $\partial^{*} \varphi(x)$ is the normal cone of $\bar{L}_{\varphi}(x)$, that is,

$$
\partial^{*} \varphi(x)=N\left(\bar{L}_{\varphi}(x), x\right):=\left\{g \in \mathbb{R}^{n}:\langle g, y-x\rangle \leq 0 \forall y \in \bar{L}_{\varphi}(x)\right\} .
$$

Furthermore, $\partial^{*} \varphi(x)$ contains nonzero vector [7]. Thus, this subdifferential is also called normal-subdifferential. 
Lemma 2.1. ([10], [14]) Assume that $\varphi: \mathbb{R}^{n} \rightarrow \mathbb{R}$ is continuous and quasiconvex. Then

$$
\partial^{*} \varphi(x) \neq \emptyset \quad \forall x \in \mathbb{R}^{n}
$$

and

$$
0 \in \partial^{*} \varphi(x) \Leftrightarrow x \in \operatorname{argmin}\left\{\varphi(y): y \in \mathbb{R}^{n}\right\} .
$$

For simplicity of notations, we let $f_{k}(x):=f\left(x^{k}, x\right)$. For the star-subdifferential we have the following results, which will be used in the sequel.

Lemma 2.2. [10] If $B(\bar{x}, \varepsilon) \subset L_{f_{k}}\left(x^{k}\right)$ for some $\bar{x} \in \mathbb{R}^{n}$ and $\varepsilon \geq 0$, then

$$
\left\langle g^{k}, x^{k}-\bar{x}\right\rangle>\varepsilon .
$$

\section{THE NORMAL SUBGRADIENT ALGORITHM AND ITS CONVERGENCE}

For presentation of the algorithm and its convergence, we make the following assumptions: Assumptions:

(A1) the solution set $S(F E P)$ is nonempty;

(A2) for every $x \in C$, the function $f(x,$.$) is continuous, quasiconvex on \mathbb{R}^{n}$, and $f(.,$.$) is$ upper semicontinuous on an open set containing $C \times C$;

(A3) the bifunction $f$ is pseudomonotone on $C$, that is,

$$
f(x, y) \geq 0 \Rightarrow f(y, x) \leq 0 \quad \forall x, y \in C,
$$

and paramonotone on $C$ with respect to the solution set $S$ of (EP), that is,

$$
x \in S, y \in C \text { and } f(x, y)=f(y, x)=0 \Rightarrow y \in S .
$$

Algorithm 3.1. Take real sequences $\left\{\alpha_{k}\right\},\left\{\lambda_{k}\right\}$ satisfying the following conditions

$$
\begin{gathered}
\alpha_{k}>0, \quad \forall k \in \mathbb{N}, \\
\sum_{k=1}^{\infty} \alpha_{k}=+\infty, \quad \sum_{k=1}^{\infty} \alpha_{k}^{2}<+\infty \\
0<\underline{\lambda} \leq \lambda_{k} \leq \bar{\lambda}<1 \quad \forall k \in \mathbb{N} .
\end{gathered}
$$

Initial Step: choose $x^{0} \in C$, let $k=0$.

Step $k(0,1 \ldots)$ : Having $x^{k} \in C$, take

$$
g^{k} \in \partial_{2}^{*} f\left(x^{k}, x^{k}\right):=\left\{g \in \mathbb{R}^{n}:\left\langle g, y-x^{k}\right\rangle<0 \quad \forall y \in L_{f_{k}}\left(x^{k}\right)\right\} .
$$

If $g^{k} \neq 0$, we normalize $g^{k}$ to obtain $\left\|g^{k}\right\|=1$. Compute

$$
y^{k}=P_{C}\left(x^{k}-\alpha_{k} g^{k}\right)
$$

and

$$
x^{k+1}=\lambda_{k} x^{k}+\left(1-\lambda_{k}\right) T\left(y^{k}\right) .
$$

If $x^{k+1}=y^{k}=x^{k}$, then STOP: $x^{k}$ is a solution.

Else update $k \longleftarrow k+1$.

Proposition 3.1. If the algorithm terminates at iteration $k$, then $x^{k}$ is a solution of (FEP). 
Proof. Let us assume that the algorithm terminates at iteration $k$. It means that $x^{k+1}=y^{k}=x^{k}$. Since $y^{k}=x^{k} \in C$, we have

$$
P_{C}\left(x^{k}-\alpha_{k} g^{k}\right)=x^{k}
$$

For every $y \in C$, it holds that

$$
\left\langle x^{k}-\alpha_{k} g^{k}-P_{C}\left(x^{k}-\alpha_{k} g^{k}\right), y-P_{C}\left(x^{k}-\alpha_{k} g^{k}\right)\right\rangle \leq 0 .
$$

This means

$$
\left\langle g^{k}, y-x^{k}\right\rangle \geq 0 \text {. }
$$

Since $g^{k} \in \partial^{*} f\left(x^{k}, x^{k}\right)$, we have that the last inequality $\left\langle g^{k}, y-x^{k}\right\rangle \geq 0, \forall y \in C$ implies $f\left(x^{k}, y\right) \geq$ $f\left(x^{k}, x^{k}\right)=0$ for every $y \in C$, which means that $x^{k}$ is a solution of (EP). Moreover, $x^{k+1}=x^{k}$. Hence $x^{k} \in F i x(T)$. This completes the proof.

The iterative sequence generated by the algorithm has the following properties.

Proposition 3.2. For every $z \in F i x(T)$, and $k \in \mathbb{N}$, the following inequality holds

$$
\begin{aligned}
\left\|x^{k+1}-z\right\|^{2} \leq & \left\|x^{k}-z\right\|^{2}+2\left(1-\lambda_{k}\right) \alpha_{k}\left\langle g^{k}, z-x^{k}\right\rangle+\left(1-\lambda_{k}\right) \alpha_{k}^{2} \\
& -\lambda_{k}\left(1-\lambda_{k}\right)\left\|x^{k}-T\left(y^{k}\right)\right\|^{2} .
\end{aligned}
$$

Proof. Let $z \in F i x(T)$. From the fact that

$$
\|t a+(1-t) b\|^{2}=t\|a\|^{2}+(1-t)\|b\|^{2}-t(1-t)\|a-b\|^{2}
$$

with $a=x^{k}-z, b=T\left(y^{k}\right)-z$ and $t=\lambda_{k}$, we have

$$
\begin{aligned}
\left\|x^{k+1}-z\right\|^{2}= & \left\|\lambda_{k} x^{k}+\left(1-\lambda_{k}\right) T\left(y^{k}\right)-z\right\|^{2} \\
\leq & \lambda_{k}\left\|x^{k}-z\right\|^{2}+\left(1-\lambda_{k}\right)\left\|T\left(y^{k}\right)-z\right\|^{2} \\
& -\lambda_{k}\left(1-\lambda_{k}\right)\left\|x^{k}-T\left(y^{k}\right)\right\|^{2} .
\end{aligned}
$$

On the other hand,

$$
\begin{aligned}
\left\|T\left(y^{k}\right)-z\right\|^{2} & \left.\leq \| y^{k}-z\right) \|^{2} \\
& =\left\|P_{C}\left(x^{k}-\alpha_{k} g^{k}\right)-z\right\|^{2} \\
& \left.\leq\left\|x^{k}-\alpha_{k} g^{k}-z\right\|^{2} \quad \text { (because } z \in C\right) \\
& =\left\|x^{k}-z\right\|^{2}-2 \alpha_{k}\left\langle g^{k}, x^{k}-z\right\rangle+\alpha_{k}^{2} .
\end{aligned}
$$

Combining this with (3.2), we obtain

$$
\left\|x^{k+1}-z\right\|^{2} \leq\left\|x^{k}-z\right\|^{2}+2\left(1-\lambda_{k}\right) \alpha_{k}\left\langle g^{k}, z-x^{k}\right\rangle+\left(1-\lambda_{k}\right) \alpha_{k}^{2}-\lambda_{k}\left(1-\lambda_{k}\right)\left\|x^{k}-T\left(y^{k}\right)\right\|^{2} .
$$

This completes the proof.

Lemma 3.1.

$$
\liminf _{k \rightarrow+\infty}\left\langle g^{k}, x^{k}-z\right\rangle \leq 0, \quad \forall z \in F i x(T) .
$$

Proof. From Proposition 3.2 and $0<\underline{\lambda} \leq \lambda_{k} \leq \bar{\lambda}<1$, it follows that

$$
2 \alpha_{k}\left\langle g^{k}, x^{k}-z\right\rangle \leq \frac{1}{1-\bar{\lambda}}\left(\left\|x^{k}-z\right\|^{2}-\left\|x^{k+1}-z\right\|^{2}\right)+\alpha_{k}^{2} .
$$


By summing up, we obtain

$$
\sum_{k=1}^{\infty} \alpha_{k}\left\langle g^{k}, x^{k}-z\right\rangle<+\infty,
$$

which together with $\sum_{k=1}^{\infty} \alpha_{k}=+\infty$ implies

$$
\liminf _{k \rightarrow+\infty}\left\langle g^{k}, x^{k}-z\right\rangle \leq 0
$$

This completes the proof.

In the following theorem, we study the convergence of our algorithm.

Theorem 3.1. If the algorithm does not terminate, then, under the assumptions (A1 )- (A3), there exists a subsequence of $\left\{x^{k}\right\}$ converges to a solution of (FEP) whenever $\left\{x^{k}\right\}$ is bounded.

Proof. We consider the following two cases.

Case 1 There exists a solution $x^{*} \in S(E P)$ and an index $k_{0}$ such that for $k \geq k_{0}$,

$$
\left\|x^{k+1}-x^{*}\right\| \leq\left\|x^{k}-x^{*}\right\| \text {. }
$$

The sequence $\left\|x_{k}-x^{*}\right\|$ is bounded below by 0 . Hence, we conclude that the sequence $\left\{\left\|x^{k}-x^{*}\right\|\right\}$ is convergent. So, the sequences $\left\{\left\|x^{k}-x^{*}\right\|\right\}$ and $\left\{x_{k}\right\}$ are bounded.

From Proposition 3.2, we have

$$
\begin{aligned}
& \lambda_{k}\left(1-\lambda_{k}\right)\left\|x^{k}-T\left(y^{k}\right)\right\|^{2} \\
\leq & \left\|x^{k}-x^{*}\right\|^{2}-\left\|x^{k+1}-x^{*}\right\|^{2}-2\left(1-\lambda_{k}\right) \alpha_{k}\left\langle g^{k}, x^{k}-x^{*}\right\rangle+\left(1-\lambda_{k}\right) \alpha_{k}^{2} .
\end{aligned}
$$

Thanks to the facts that $\left\|g^{k}\right\|=1$ and $\left\{\left\|x^{k}-x^{*}\right\|\right\}$ is bounded, the right hand side of the above inequality goes to 0 when $k$ goes to infinity. Since $0<\underline{\lambda} \leq \lambda_{k} \leq \bar{\lambda}<1$, we obtain

$$
\lim _{k \rightarrow \infty}\left\|x^{k}-T\left(y^{k}\right)\right\|=0
$$

In addition, we have

$$
\begin{aligned}
\left\|x^{k}-T\left(x^{k}\right)\right\| & \leq\left\|x^{k}-T\left(y^{k}\right)\right\|+\left\|T\left(y^{k}\right)-T\left(x^{k}\right)\right\| \\
& \leq\left\|x^{k}-T\left(y^{k}\right)\right\|+\left\|y^{k}-x^{k}\right\| \\
& =\left\|x^{k}-T\left(y^{k}\right)\right\|+\left\|P_{C}\left(x^{k}-\alpha_{k} g^{k}\right)-x^{k}\right\| \\
& \leq\left\|x^{k}-T\left(y^{k}\right)\right\|+\left\|x^{k}-\alpha_{k} g^{k}-x^{k}\right\| \\
& \leq\left\|x^{k}-T\left(y^{k}\right)\right\|+\alpha_{k} .
\end{aligned}
$$

Since $\lim _{k \rightarrow \infty} \alpha_{k}=0$, we have

$$
\lim _{k \rightarrow \infty}\left\|x^{k}-T\left(x^{k}\right)\right\|=0
$$

Thanks to Lemma 3.1, we have

$$
\liminf _{k \rightarrow \infty}\left\langle g^{k}, x^{k}-x^{*}\right\rangle \leq 0 .
$$

Let $\left\{x^{k_{i}}\right\}$ be a subsequence of $\left\{x^{k}\right\}$ such that

$$
\lim _{i \rightarrow \infty}\left\langle g^{k_{i}}, x^{k_{i}}-x^{*}\right\rangle=\liminf _{k \rightarrow \infty}\left\langle g^{k}, x^{k}-z\right\rangle .
$$


Since $\left\{x^{k}\right\}$ is bounded, we have that $\left\{x^{k_{i}}\right\}$ is bounded too. Let $\bar{x}$ be a limit point of $\left\{x^{k_{i}}\right\}$. Without loss of generality, we may assume that

$$
\lim _{i \rightarrow \infty} x^{k_{i}}=\bar{x}
$$

It is easy to see that

$$
\begin{aligned}
\left\|x^{k_{i}}-T(\bar{x})\right\| & \leq\left\|x^{k_{i}}-T\left(x^{k_{i}}\right)\right\|+\left\|T(\bar{x})-T\left(x^{k_{i}}\right)\right\| \\
& \leq\left\|x^{k_{i}}-T\left(x^{k_{i}}\right)\right\|+\left\|\bar{x}-x^{k_{i}}\right\| .
\end{aligned}
$$

Thanks to (3.5) and (3.7), we have

$$
\lim _{i \rightarrow \infty} x^{k_{i}}=T(\bar{x}) \text {. }
$$

Combining this with (3.7), we see that $T(\bar{x})=\bar{x}$, which implies $\bar{x} \in F i x(T)$.

In addition, since $x^{*}$ is a solution of the equilibrium problem, from the pseudomonotonicity of $f$ on $C$, we have $f\left(\bar{x}, x^{*}\right) \leq 0$. Moreover, $f\left(\bar{x}, x^{*}\right)=0$. Indeed, let us assume that $f\left(\bar{x}, x^{*}\right) \leq-a$. for some positive number $a$. From the upper semicontinuity of $f$ on an open set containng $C \times C$, there exist positive numbers $\varepsilon_{1}, \varepsilon_{2}$ such that, for any $x \in B\left(\bar{x}, \varepsilon_{1}\right), y \in B\left(x^{*}, \varepsilon_{2}\right), f(x, y) \leq-\frac{a}{2}$.

In addition, $\lim _{i \rightarrow \infty} x^{k_{i}}=\bar{x}$ implies that there exist $i_{0}$ such that, for $i \geq i_{0}, x^{k_{i}}$ belongs to $B\left(\bar{x}, \varepsilon_{1}\right)$. So, for $i \geq i_{0}$ and $y \in B\left(x^{*}, \varepsilon_{2}\right)$, we have

$$
f\left(x^{k_{i}}, y\right) \leq-\frac{a}{2}
$$

which implies that $B\left(x^{*}, \varepsilon_{2}\right) \subset L_{f_{k_{i}}}\left(x^{k_{i}}\right)$. On the other hand, $g^{k_{i}} \neq 0$ because if $g^{k_{i}}=0$, then $f\left(x^{k_{i}}, y\right) \geq f\left(x^{k_{i}}, x^{k_{i}}\right)=0$ for every $y \in \mathbb{R}^{n}$, which contradicts (3.10). By Lemma 2.2, for $i \geq$ $i_{0}$, it holds that $\left\langle g^{k_{i}}, x^{k_{i}}-x^{*}\right\rangle>\varepsilon_{2}$, which contradicts (3.6). Thus, $\bar{x} \in C$ and $f\left(\bar{x}, x^{*}\right)=0$. Again, by using the pseudomonotonicity, we obtain $f\left(x^{*}, \bar{x}\right)=0$. Then, it follows from the paramonotonicity of $f$ that $\bar{x}$ is a solution of (EP). So $\bar{x}$ is a solution of (FEP)

Case 2 For any solution $x^{*}$ of (EP), there exists a subsequence $\left\{x_{k_{i}}\right\}$ of $\left\{x^{k}\right\}$ satisfying

$$
\left\|x^{k_{i}}-x^{*}\right\|<\left\|x^{k_{i}+1}-x^{*}\right\| .
$$

Thanks to Proposition 3.2, we have

$$
\begin{aligned}
& \left.\lambda_{k_{i}}\left(1-\lambda_{k_{i}}\right) \| x^{k_{i}}-T\left(y^{k_{i}}\right)\right) \|^{2} \\
\leq & \left\|x^{k_{i}}-x^{*}\right\|^{2}-\left\|x^{k_{i}+1}-x^{*}\right\|^{2}-2\left(1-\lambda_{k_{i}}\right) \alpha_{k_{i}}\left\langle g^{k_{i}}, x^{k_{i}}-x^{*}\right\rangle+\left(1-\lambda_{k_{i}}\right) \alpha_{k_{i}}^{2} . \\
\leq & -2\left(1-\lambda_{k_{i}}\right) \alpha_{k_{i}}\left\langle g^{k_{i}}, x^{k_{i}}-x^{*}\right\rangle+\left(1-\lambda_{k_{i}}\right) \alpha_{k_{i}}^{2} .
\end{aligned}
$$

It follows that

$$
\lim _{i \rightarrow \infty}\left\|x^{k_{i}}-T\left(y^{k_{i}}\right)\right\|=0
$$

Also, it follows from (3.11) that

and

$$
\left\langle g^{k_{i}}, x^{k_{i}}-x^{*}\right\rangle \leq \frac{\alpha_{k_{i}}}{2}
$$

$$
\limsup _{i \rightarrow \infty}\left\langle g^{k_{i}}, x^{k_{i}}-x^{*}\right\rangle \leq 0 .
$$

Now by the same argument as in Case 1, we see that every limit point of sequence $\left\{x_{k_{i}}\right\}$ belongs to the solution set $S(F E P)$. This completes the proof. 


\section{Illustrative Models}

Many problems can be formulated in the form of problem (FEP). Let us consider the following two ones.

\section{Common solutions of equilibrium and variational inequality problems.}

Consider the problem of finding a common solution of an equilibrium problem and a variational inequality, which can be formulated as

$$
\text { finding } x^{*} \in C: f\left(x^{*}, y\right) \geq 0,\left\langle F\left(x^{*}\right), y-x^{*}\right\rangle \geq 0, \quad \forall y \in C,
$$

where, as before, $C$ is a closed convex subset of a real Hilbert space $\mathscr{H}, f: C \times C \rightarrow \mathscr{R}$ and $F: \mathscr{H} \rightarrow \mathscr{H}$. Suppose that $F$ is $\delta$-cocoercive (strongly reverse monotone) on $C$, that is,

$$
\langle F(x)-F(y), x-y\rangle \geq \delta\|F(x)-F(y)\|^{2} \forall x, y \in C
$$

with $\delta>0$. This concept was introduced in [20] (see [8] for more details). The variational inequality with the cost operator being strongly reverse monotone can be formulated equivalently to a nonexpansive fixed point problem as stated in the following proposition.

Proposition 4.1. Let $F$ be $\delta$-strongly reverse monotone on $C$. Then the solution set of the variational inequality problem

$$
\text { find } x^{*} \in C:\left\langle F\left(x^{*}\right), y-x^{*}\right\rangle \geq 0, \quad \forall y \in C
$$

coincides with the fixed-point set of the nonexpansive mapping $h(x):=P_{C}(x-\lambda F(x))$ whenever $\lambda>1 / 2 \delta$.

Proof. First, we observe that $x$ is a solution of the variational inequality problem if and only if it is a fixed point of the mapping $h(x):=P_{C}(x-\lambda F(x))$ with any $\lambda>0$. Then by using the nonexpansiveness of the metric projection, we can easily show that

$$
\|h(x)-h(y)\|^{2} \leq \| x-y-\frac{1}{\lambda}\left((F(x)-F(y)) \|^{2} .\right.
$$

However,

$$
\| x-y-\frac{1}{\lambda}\left((F(x)-F(y))\left\|^{2}=\right\| x-y\left\|^{2}-\frac{2}{\lambda}\langle x-y, F(x)-F(y)\rangle+\frac{1}{\lambda^{2}}\right\| F(x)-F(y) \|^{2} .\right.
$$

Since $F$ is $\delta$-strongly reverse monotone on $C$ and $\lambda \geq 1 / 2 \delta$, we have

$$
\left\|x-y-\frac{1}{\lambda}(F(x)-F(y))\right\|^{2} \leq\|x-y\|^{2}, \quad \forall x, y \in C,
$$

which together with (4.1) yields that $h$ is nonexpansive on $C$.

\section{A practical model}

Suppose that a firm products $n$-types of electricity. Let $x=\left(x_{1}, \ldots, x_{n}\right)^{T}$ be the vector where the component $x_{j}$ denotes the quantity of the type $j$. Let us denote by $u$ the utility function. Suppose that the production must satisfy certain condition given as $x \in B$. For example, $B$ is a box defined as $B:=\{x: 0<L \leq x \leq U<\infty\}$ with $L, U$ being given vectors in $\mathscr{R}^{n}$. In practice, the level of the production of each type electricity should satisfy a certain ratio, for example, the ratio of hydroelectricity $x_{1}$ and the total production $m:=\sum_{j} x_{j}$ of all other electricity-types should be restricted in a given percent. In general, this condition can be given as $x \in S$ with $S$ being a polyhedral convex set, for example, $S:=\left\{x=\left(x_{1}, \ldots, x_{j}, . ., x_{n}\right)^{T} \in \mathscr{R}^{n}, 0 \leq \frac{x_{j}}{m} \leq \tau, j=1, . . n\right\}$. 
So, if $T(x):=P_{S}(x)$, then $T$ is nonexpansive on $\mathscr{H}$. Now, we define $f(x, y):=u(x)-u(y)$. Then the model can be formulated in the form of Problem (FEP). Clearly, $f$ is paramonotone and $f(x,$.$) is quasiconvex if u$ is quasiconcane, for example, $u$ is an affine fractional or a CobbDouglas production function (often in economics).

\section{CONCLUSION}

We proposed the algorithm for finding a common solution of the paramonotone quasiconvex equilibrium and the nonexpansive fixed point problem. Due to the quasiconvexity of the bifunction involved, the regularization as well as extragradient methods that commonly used in the subject may fail due to the quasiconvexity since the sum of a quasiconvex function and a convex function may not be convex even not quasiconvex. The proposed algorithm is a combination of the projection method and the Krasnoselskii-Mann iteration scheme. We established the convergence of the algorithm and also provide the illustrative practical models.

\section{Acknowledgement}

This research was funded by Vietnam National Foundation for Science and Technology Development (NAFOSTED) under grant number 101.01-2020.06.

\section{REFERENCES}

[1] P. N. Anh, L.D. Muu: A hybrid subgradient algorithm for nonexpansive mapping and equilibrium problems, Optim. Lett. 8 (2014), 727-738.

[2] P.N. Anh, A hybrid extragradient method extended to fixed point problems and equilibrium problems, Optimization 62 (2011), 271-283.

[3] E. Blum, W. Oettli, From optimization and variational inequalities to equilibrium problems, Math. Stud. 62 (1994), 127-169.

[4] G. Bigi, M. Castellani, M. Pappalardo, M. Passacantando, Nonlinear Programming Techniques for Equilibria, Springer, Switzerland, 2019.

[5] A. Cegielski, Iterative methods for fixed point problems in Hilbert spaces, Springer, 2013.

[6] K. Fan, A minimax inequality and applications. In: Shisha O. (Ed.): Inequalities, pp. 103-113, Academic Press, New York, 1972.

[7] H.P. Greenberg, W.P. Pierskalla, Quasi-conjugate functions and surogate duality, Cahiers Centre Études Recherche Oper. 15 (1973), 437-448.

[8] E.G. Golshtein, N.V. Tretyakov, Modifiled Lagrangians and Monotone Maps in Optimization, Wiley, New York, 1996.

[9] G. Kassay, V. Rădulescu, Equilibrium problems and applications, Academic Press, 2018. https://doi.org/10.1016/C2015-0-06685-0.

[10] K.C. Kiwiel, Convergence and efficiency of subgradient methods for quasiconvex minimization, Math. Program. Ser. A 90 (2001), 1-25.

[11] L.D. Muu, W. Oettli, Convergence of an adaptive penalty scheme for finding constrained equilibria, Nonlinear Anal 18 (1992), 1159-1166.

[12] H. Nikaido, K. Isoda, Note on noncooperative convex games, Pac. J. Math. 5 (1955), 807-815.

[13] F.U. Ogbuisi, Popov subgradient extragradient algorithm for pseudomonotone equilibrium problems in Banach spaces, J. Nonlinear Funct. Anal. 2019 (2019), Article ID 44.

[14] J.-P. Penot, C. Zalinescu, Elements of quasiconvex subdifferential calculus, J. Convex Anal. 7 (2000), 243269.

[15] J.-P. Penot, Are generalized derivatives useful for generalized convex functions? In: J.-P. Crouzeix, J.E. Martinez-Legaz and M. Volle (Ed.), Generalized convexity, Generalized Monotonicity: Recent Results, pp. 3-59, Kluwer Academic Publishers, Dordrecht, The Netherlands, 1998. 
[16] X. Qin, Y.J. Cho, S.M. Kang, Convergence theorems of common elements for equilibrium problems and fixed point problems in Banach spaces, J. Comput. Appl. Math. 225 (2009), 20-30.

[17] A. Tada, W. Takahashi, Weak and Strong Convergence Theorems for a Nonexpansive Mapping and an Equilibrium Problem, J Optim Theory Appl. 133 (2007), 359-370.

[18] S. Takahashi W. Takahashi, Viscosity approximation methods for equilibrium problems and fixed point problems in Hilbert spaces, J. Math. Anal. Appl. 331 (2007), 506-515/

[19] L.H. Yen, L.D. Muu, A subgradient method for equilibrium problems involving quasiconvex bifunction, Oper. Res. Lett 48 (2020), 579-583.

[20] D. Zhu, P. Marcotte, A new class of generalized monotonicity, J. Optim. Theory Appl. 87 (1995), 457-471. 